\title{
CARDIAC OUTPUT IN PNEUMONIA IN THE DOG
}

\author{
BY TINSLEY R. HARRISON AND ALFRED BLALOCK
}

(From the Departments of Medicine and Surgery, Vanderbilt University, School of Medicine)

(Received for publication April 5, 1926)

\section{INTRODUCTION}

The condition of the circulation of patients suffering from pneumonia is always of definite concern to the physician. An attempt is commonly made to discover the onset of signs of cardiac failure, and to evaluate the degree of failure when these signs appear, as circulatory failure is an important factor leading to a fatal outcome in many cases of pneumonia. The mechanism of cardiac failure in pneumonia, however, is not well understood, and no satisfactory explanation has been offered for the relation of disturbance of the circulation to the pulmonary lesions or to the effects of the organisms responsible for the disease. Doubt has been cast on the assumption that failure of the heart is the result of the direct action of the toxins of the infecting organism on the myocardium. Newburgh and Porter (1) found that strips of muscle from the cardiac ventricles of dogs dying of pneumonia contracted as well as similar strips from normal dogs when both were perfused with normal blood. When "pneumonic blood" was used as a perfusate the heart muscle of pneumonic dogs contracted better than the muscle of normal dogs. These results led Newburgh and Porter to assume the existence in pneumonic blood of a toxin to which the animals dying of the disease had become relatively immune.

From a review of the literature and from their own studies Cohn and Jamieson (2) concluded that significant alterations in the heart muscle are not generally found in pneumonia.

The question of the effect of the pulmonary lesion on the circulation has received but scant attention. On general principles it is evident that if the functional efficiency of the lungs is lowered so that the blood is supplied with less than its normal quota of oxygen in its 
passage through the pulmonary circulation, an increased blood flow is necessary to meet the oxygen requirements of the body. For this reason the task of the heart is increased when pulmonary consolidation interferes with the gas exchange in the lungs. The augmented demand of the body for oxygen which accompanies fever adds a further burden on the heart, so that in pneumonia the demands on the circulation may be especially heavy, and may at times surpass the ability of the circulation to respond. Then signs of circulatory failure appear.

Few investigations of the circulation in pneumonia from the point of view of its respiratory functions have appeared. Stadie (3) studied the arterial and venous oxygen contents of the blood of patients with pneumonia. He stated: "The oxygen consumption, i.e., difference between arterial and venous contents was within normal limits, indicating that the cardiac output was not diminished." His figures show a low rather than a high arterio-venous difference. Assuming an increased rate of tissue oxidation, such as is usually found in fevers, one is led to believe from Stadie's data that cardiac output was probably actually increased.

Leegaard (4) recently published preliminary observations on cardiac output in rabbits after pneumonia had been induced by the intratracheal injection of pneumococci. He found a 50 to 100 per cent increase in the minute output of the heart.

Our interest in the problem followed the accidental finding of a high cardiac output in a supposedly normal dog, which at autopsy was found to have extensive broncho-pneumonia. Since then eightyseven determinations of blood flow have been made on twenty-four additional dogs. In this paper the various factors causing variations in the output of the heart in pneumonia are analyzed and discussed. Observations regarding other factors bearing on the problem of variations in the cardiac output will be considered in subsequent papers.

\section{METHODS}

Three different types of pneumonia have been studied:

1. Spontaneous pneumonia. Measurements of the cardiac output were made on a number of stock animals showing signs of severe distemper, emaciation, and fever. After two or more determinations the animals were sacrificed. Three of the group were found at necropsy to have pneumonia. 
2. Post-operative pneumonia. The cardiac output was studied in nine dogs in which pneumonia developed following various operations-most frequently after thoracotomies.

3. Induced pneumonia. Intrabronchial injection of pneumococci or Friedlander bacilli was done in thirteen dogs.

In the animals of the first two groups control studies were not available, while in the third group the cardiac output was measured before and after the injection of organisms. Difficulty was encountered in the study of the third group in the regulation of the dosage of the organisms injected, as dosage was either so large as to kill the animal in a few hours, or so small as to produce no appreciable effect. Of thirteen dogs inoculated only six lived long enough to develop clinical evidence of pneumonia. When the dose was small the animals appeared to recover very rapidly. With large doses death occurred apparently from an overwhelming infection, without the characteristic picture of pneumonia. From our results we conclude that only one of the dogs with induced pneumonia had the disease long enough to develop a picture simulating the more chronic pneumonia of the other groups.

Most of the animals received morphine 0.006 gram per kilo, 30 to 60 minutes before each determination. Several determinations were done without morphine and the results were similar to those found in the dogs receiving the drug. This indicates that the effect of morphine on cardiac output was negligible in these experiments. Marshall (5) has found that morphine had no appreciable effect on cardiac output of the normal dog, and Blalock, Harrison, and Wilson (6) using morphinized dogs found results for blood flow only slightly higher than the result of Marshall on unanesthetized trained dogs.

The oxygen consumption was determined by means of a Benedict spirometer with the graphic recording device. In earlier experiments a rubber bladder was made air tight over the animal's head in order to obtain an accurate measurement of the respiration. In later experiments a paraffined plaster of paris mask was fitted to the dog's nose and made air tight with plasticine.

Venous blood was obtained by puncture of the right ventricle; arterial blood from the left ventricle or the femoral artery. Two samples of blood were usually drawn from the right ventricle in order to guard against errors arising from the possible mixture of arterial blood with the venous blood. The usual precautions against contact with air were followed. The arterial and venous oxygen contents were determined with the Van Slyke (7) long bore, constant pressure apparatus in the first experiments; the later determinations were made with the Van Slyke-Neill (8) constant volume apparatus. Oxygen capacity was also determined in some instances.

The cardiac output per minute was calculated according to the Fick formula;

cc. oxygen consumed per minute
cc. oxygen taken up by 1 cc. of
blood in passing through the lungs


The hydrogen ion concentration of the blood was determined in a few instances, Hawkins (9) modification of Cullen's (10) colorimetric method being used.

When the dogs struggled, or when a reasonable suspicion was entertained that either venous or arterial blood was "mixed" the experiment was discarded.

Duplicate five-, or ten-minute periods were used in determining the oxygen consumption. Check determinations were made of the oxygen content of the blood. Single determinations of oxygen capacity were considered sufficiently reliable.

Most of the animals died spontaneously. A few were killed either intentionally or by the production of hemopericardium. The gross anatomical findings were confirmed by microscopic examination of the tissues. The output of the heart per minute is for the sake of comparison divided by the kilograms of body weight of each animal. The output per minute is also divided by the number of heart beats per minute in order to obtain the average cardiac output per beat.

\section{RESULTS}

The details of the results of each experiment are given in the accompanying tables.

The results of the experiments on dogs with spontaneous pneumonia and on dogs developing post-operative pneumonia are similar and are therefore considered together. In the eleven animals constituting these two series the cardiac output varied between 200 and $400 \mathrm{cc}$. of blood per minute per kilo of body weight, and averaged $274 \mathrm{cc}$. These figures appear in sharp contrast to those obtained from normal dogs, in which the cardiac output has been found to be from 100 to 200 cc. per minute per kilo. In a series of 18 normal dogs the cardiac output averaged $167 \mathrm{cc}$. per minute per kilo. Thus it is evident that the dogs with spontaneous and post-operative pneumonia had a striking increase in the output of the heart per minute.

The series of animals in which pneumonia was induced by the intrabronchial injections of bacteria gave results regarding the output of the heart which are quite different from those obtained in the other two groups (tables 11 to 14). In this series of six dogs, with one exception, no significant change in the cardiac output was observed after pneumonia was induced when compared with the measurements made before the organisms were inoculated into the bronchi. In one animal a definite increase in the output of the heart was observed. The average results of the entire series are as follows:

1. Before inoculation (eleven determinations) $\ldots \ldots \ldots \ldots \ldots \ldots \ldots \ldots .170$

2. After inoculation (thirteen determinations) $\ldots \ldots \ldots \ldots \ldots \ldots \ldots \ldots . \ldots . \ldots 19$

3. After inoculation (twelve determinations) $\ldots \ldots \ldots \ldots \ldots \ldots \ldots \ldots$ 
TABLE 1

- Pneumonia following operation

Animal I2. Weight varied 4.6 to 5.9 kilos

\begin{tabular}{|c|c|c|c|c|c|c|c|c|c|c|c|}
\hline$\stackrel{\mathscr{u}}{\mathrm{J}}$ & 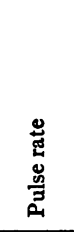 & 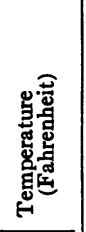 & 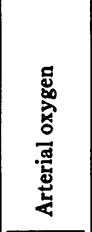 & 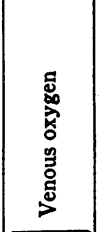 & 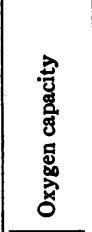 & 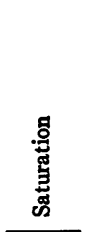 & 뗨 & 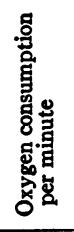 & 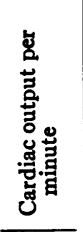 & 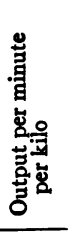 & 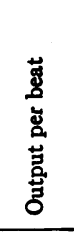 \\
\hline & $\begin{array}{c}\text { per } \\
\text { minute }\end{array}$ & & $\begin{array}{l}\text { oolumes } \\
\text { per cent }\end{array}$ & $\begin{array}{l}\text { volumes } \\
\text { per cent }\end{array}$ & $\begin{array}{l}\text { oolumes } \\
\text { per cent }\end{array}$ & per cent & & $c c$. & $c c$. & $c c$. & $c c$. \\
\hline $5-10$ & 68 & 100.1 & 15.1 & 12.7 & 17.4 & 86.8 & & 36.4 & 1,517 & 330 & 22.3 \\
\hline 5-11 & 56 & 100.5 & 14.4 & 10.5 & 17.1 & 84.2 & & 52.7 & 1,351 & 294 & 24.0 \\
\hline $5-23$ & 54 & 99.8 & 15.4 & 11.4 & & & 7.28 & 38.2 & 1,151 & 250 & 21.3 \\
\hline $5-26$ & 62 & 98.0 & 14.6 & 10.8 & & & & 37.6 & 989 & 219 & 16.0 \\
\hline $6-17$ & 64 & & 19.6 & 13.7 & & & & 43.7 & 741 & 148 & 11.6 \\
\hline $10-3$ & 60 & 99.0 & 22.02 & 17.31 & & & & 44.7 & 949 & 160 & 15.8 \\
\hline $10-22$ & 78 & 102.0 & 18.27 & 11.49 & & & & 56.4 & 725 & 123 & 9.0 \\
\hline
\end{tabular}

(Figures in italics denote determinations done when animal was apparently normal; other figures denote determinations when pneumonia was thought to be present.)

Protocol: April 21, 1925, pericardotomy performed. May 7, dog looks sick. May 11, chest X-rayed, thought to show pneumonia. June 23, dog seems very much improved, gaining weight. October 10, looks well October 20, listless and febrile, without evidence of distemper or pneumonia. (Morphine 0.04 gram given before each determination.)

TABLE 2

Pneumonia following operation

Animal C8. Weight varied 8.0 to 7.7 kilos.

\begin{tabular}{|c|c|c|c|c|c|c|c|c|c|c|}
\hline $\begin{array}{l}\stackrel{\Xi}{\Xi ّ} \\
\text { ه }\end{array}$ & 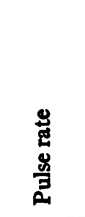 & 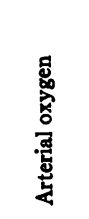 & 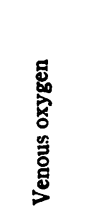 & 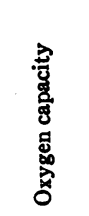 & 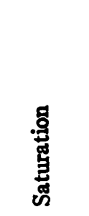 & 圈 & 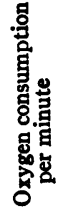 & 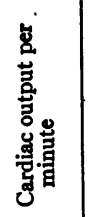 & 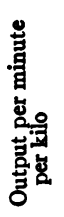 & 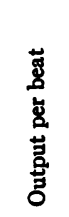 \\
\hline & $\begin{array}{c}\text { per } \\
\text { minute }\end{array}$ & $\begin{array}{l}\text { volumes } \\
\text { per cent }\end{array}$ & $\begin{array}{l}\text { volumes } \\
\text { per cent }\end{array}$ & $\begin{array}{l}\text { volumes } \\
\text { per cent }\end{array}$ & per cent & & $c c$. & $c c$. & $c c$. & cc. \\
\hline $12-9-24$ & 45 & 20.4 & 15.4 & 21.9 & 93.1 & & 48.1 & 962 & 120 & 21.4 \\
\hline $1-4-25$ & 56 & 17.8 & 15.6 & 20.2 & 88.1 & 7.33 & 48.1 & 2,187 & 284 & 39.0 \\
\hline
\end{tabular}

(Figures in italics denote determinations done when animal was apparently normal; other figures denote determinations when pneumonia was thought to be present.)

Protocol: December 9, 1924, tracheotomy. December 16, dog has distemper. January 4,1925 , distemper seems worse, morphia 0.06 gram before determination. Extensive bilateral pneumonia found at autopsy. 
If the one experiment in which an increased cardiac output following inoculation is omitted from the series, the average results indicate that no increase in the minute output of the heart took place after inoculation.

TABLE 3

Pneumonia following operation

Animal I3. Weight 5.1 kilos

\begin{tabular}{c|c|c|c|c|c|c|c|c}
\hline Date & Pulse rate & $\begin{array}{c}\text { Temper- } \\
\text { ature } \\
\text { (Fahren- } \\
\text { heit) }\end{array}$ & $\begin{array}{c}\text { Arterial } \\
\text { oxygen }\end{array}$ & $\begin{array}{c}\text { Venous } \\
\text { oxygen }\end{array}$ & $\begin{array}{c}\text { Oxygen } \\
\text { consump- } \\
\text { tion per } \\
\text { minute }\end{array}$ & $\begin{array}{c}\text { Cardiac } \\
\text { output } \\
\text { per } \\
\text { minute }\end{array}$ & $\begin{array}{c}\text { Output per } \\
\text { minute } \\
\text { per kilo }\end{array}$ & $\begin{array}{c}\text { Output } \\
\text { per beat }\end{array}$ \\
\hline & per minute & & $\begin{array}{c}\text { volumes } \\
\text { per cent }\end{array}$ & $\begin{array}{c}\text { volumes } \\
\text { per cent }\end{array}$ & $c c$. & $c c$. & cc. & cc. \\
$5-10$ & 98 & 104.8 & 14.5 & 7.1 & 50.3 & 679 & 133 & 7.1 \\
$5-11$ & 122 & 102.0 & 13.2 & 7.8 & 48.9 & 906 & 177 & 7.4 \\
\hline
\end{tabular}

Protocol: April 25, 1925, pericardotomy. During the following week animal was listless and refused to eat. May 11, seems much sicker. May 12, extensive bronchopneumonia and adhesive pericarditis found at autopsy.

TABLE 4

Pneumonia following operation

Animal I4. Weight varied 6.4 to 5.6 kilos

\begin{tabular}{|c|c|c|c|c|c|c|c|c|c|c|c|}
\hline 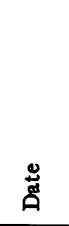 & 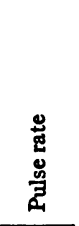 & 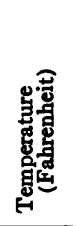 & 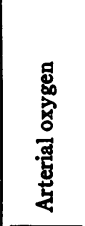 & 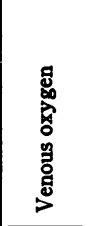 & 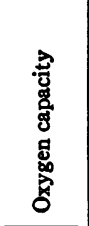 & 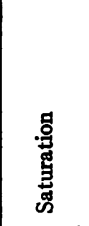 & 思 & 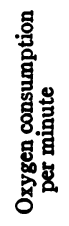 & 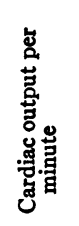 & 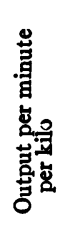 & 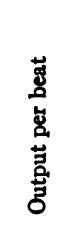 \\
\hline & $\underset{\text { per }}{\text { minute }}$ & & $\begin{array}{l}\text { oolumes } \\
\text { per cent }\end{array}$ & $\begin{array}{l}\text { volumes } \\
\text { per cent }\end{array}$ & $\begin{array}{l}\text { volumes } \\
\text { per cent }\end{array}$ & per cent & & $c c$. & $c c$. & $c c$. & $c c$. \\
\hline $5-21$ & 55 & 103.6 & 16.4 & 12.7 & 20.2 & 81.2 & & 74.0 & 2,000 & 313 & 36.3 \\
\hline 5-25 & 82 & 102.2 & 7.7 & 3.8 & 12.3 & 62.6 & & 63.0 & 1,620 & 270 & 19.7 \\
\hline 5-27 & 63 & 101.5 & 8.0 & 3.0 & 11.8 & 67.8 & 7.31 & 60.1 & 1,205 & 215 & 19.1 \\
\hline
\end{tabular}

Protocol: April 28, 1925, nerve suture operation performed. May 12, distemper noticed. Animal appeared quite sick and began to lose weight. May 21, looks very sick. May 25, continues to appear sick. X-ray shows extensive consolidation on both sides. May 27, moribund. May 28, found dead. Complete consolidation of left lung and patchy consolidation of right lung found at autopsy. (Morphine 0.04 gram given before each determination.)

The rectal temperature was slightly elevated in most, but not all, of the dogs with pneumonia. The pulse rate was sometimesmoderately increased, but pulse rates are of little significance in morphinized 
TABLE 5

Pneumonia following operation

\begin{tabular}{|c|c|c|c|c|c|c|c|}
\hline Date & Pulse rate & $\begin{array}{l}\text { Arterial } \\
\text { oxygen }\end{array}$ & $\begin{array}{l}\text { Venous } \\
\text { oxygen }\end{array}$ & $\begin{array}{l}\text { Oxygen } \\
\text { consump- } \\
\text { tion per } \\
\text { minute }\end{array}$ & $\begin{array}{l}\text { Cardiac } \\
\text { output per } \\
\text { minute }\end{array}$ & $\begin{array}{l}\text { Output per } \\
\text { minute } \\
\text { per kilo }\end{array}$ & $\begin{array}{l}\text { Output } \\
\text { per beat }\end{array}$ \\
\hline
\end{tabular}

Animal 5. Weight 6 kilos.

\begin{tabular}{c|r|r|r|r|r|r|r|r}
\hline & per minute & $\begin{array}{r}\text { volumes } \\
\text { per cent }\end{array}$ & $\begin{array}{r}\text { volumes } \\
\text { per cent }\end{array}$ & cc. & cc. & cc. & $c c$. \\
$4-5$ & 76 & 19.2 & 16.7 & 56.0 & 2,240 & 373 & 29.4 \\
\hline
\end{tabular}

Animal 6. Weight 5.3 kilos

\begin{tabular}{l|l|l|l|l|l|l|l}
\hline $4-12$ & 56 & 16.5 & 11.1 & 41.2 & 763 & 143 & 13.6 \\
\hline
\end{tabular}

Protocol: Animal No. 5. March 7, 1925, pericardotomy. April 2, animal appears sick. April 5, very weak, unable to stand. April 6, dead. Lungs showed extensive bilateral broncho-pneumonia.

Protocol: Animal No. 6. March 14, 1925, pericardotomy. April 8, animal appears quite sick. April 12, seems improved. April 16, animal dead. Many pericardial adhesions and a few scattered patches of broncho-pneumonia found at autopsy. (Given 0.04 gram of morphine before determinations.)

TABLE 6

Pneumonia following operation

Animal V31. Weight 6.7 kilos

\begin{tabular}{|c|c|c|c|c|c|c|c|c|c|c|}
\hline Date & $\begin{array}{l}\text { 岕 } \\
\text { 巴ّ } \\
\text { 苞 }\end{array}$ & 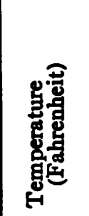 & 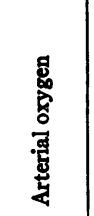 & $\begin{array}{l}\text { : } \\
0 \\
0 \\
0 \\
0 \\
0 \\
0 \\
0 \\
0\end{array}$ & 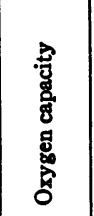 & 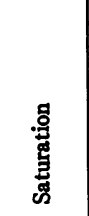 & 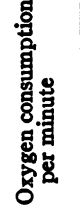 & 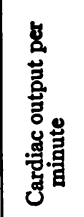 & 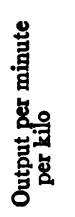 & 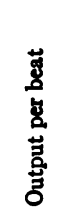 \\
\hline & $\begin{array}{c}\text { per } \\
\text { minute }\end{array}$ & & $\begin{array}{l}\text { volumes } \\
\text { per cent }\end{array}$ & $\begin{array}{l}\text { volumes } \\
\text { per cent }\end{array}$ & $\begin{array}{l}\text { oolumes } \\
\text { per cent }\end{array}$ & per cent & $c c$. & $c c$. & cc. & $c c$. \\
\hline 2-20 1 p.m. & 48 & 97.0 & 20.68 & 14.99 & 21.5 & 96.2 & 45.6 & 801 & 119 & 16.6 \\
\hline 2-20 3 p.m. & 52 & 97.4 & 18.49 & 12.58 & 20.23 & 91.4 & 46.9 & 808 & 120 & 15.5 \\
\hline 2-22 9 a.m. & 62 & 100.2 & 12.36 & 9.12 & $|16.44|$ & 75.2 & 57.6 & 1,777 & 278 & 28.6 \\
\hline 2-22 11 a.m. & 56 & & 12.65 & 8.10 & 16.12 & 78.5 & 56.9 & 1,248 & 192 & 22.0 \\
\hline
\end{tabular}

(Figures in italics denote determinations done when animal was apparently normal; other figures denote determinations when pneumonia was thought to be present.)

Protocol: February 20, 1925, tracheotomy performed. Two control determinations on this date. February 22, appears weak, neck wound infected. February 23, animal killed. Complete consolidation left upper lobe and patchy consolidation elsewhere found at autopsy. 
TABLE 7

Spontaneous preumonia

Animal M2. Weight varied 6.2 to 6 kilos

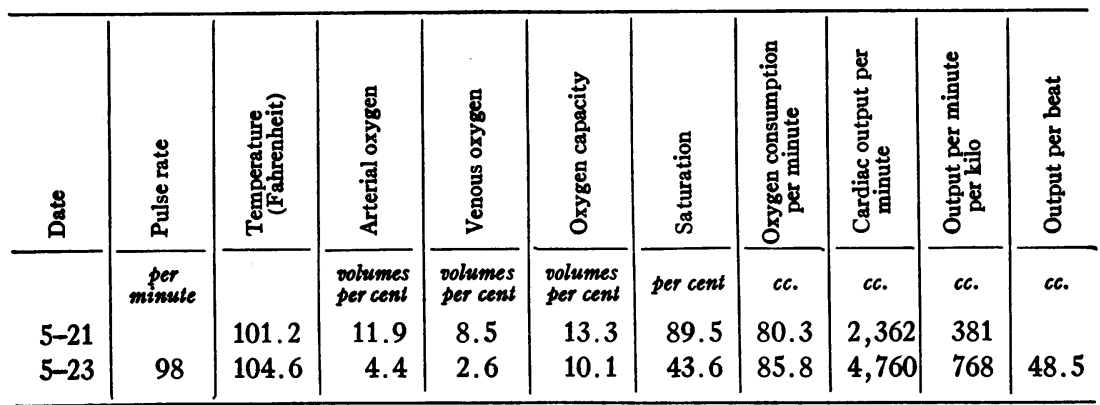

Protocol: May 7, 1925, distemper first noticed. May 21, dog has lost a great deal of weight. Profuse discharge from nose and eyes. First determination on blood flow. May 23, unable to stand. Second determination. May 24, found dead. Complete consolidation right lower lobe and extensive patches of pneumonia throughout the rest of the lungs were found at autopsy. (Morphine 0.03 gram given before each determination.)

TABLE 8

Spontaneous pneumonia

Animal V7. Weight varied 7.8 to 6.6 kilos

\begin{tabular}{|c|c|c|c|c|c|c|c|c|c|c|c|}
\hline 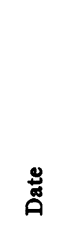 & $\begin{array}{l}\text { 苞 } \\
\text { 岁 } \\
\text { 节 }\end{array}$ & 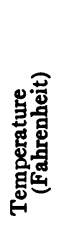 & 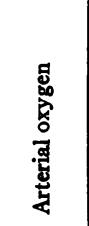 & 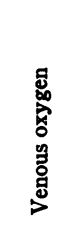 & 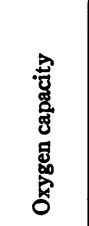 & 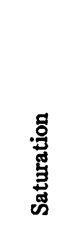 & 窝 & 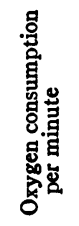 & 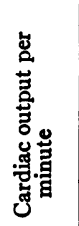 & 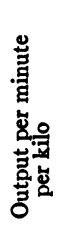 & 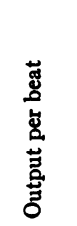 \\
\hline & $\begin{array}{c}\text { per } \\
\text { minute }\end{array}$ & & $\begin{array}{l}\text { volumes } \\
\text { per cent }\end{array}$ & $\begin{array}{l}\text { volumes } \\
\text { per cent }\end{array}$ & $\begin{array}{l}\text { volumes } \\
\text { per cent }\end{array}$ & per cent & & $c c$. & cc. & cc. & $c c$. \\
\hline $10-27$ & 126 & 101 & 8.93 & 4.80 & 10.74 & 83.1 & & 112.8 & 2,731 & 350 & 21.6 \\
\hline $10-28$ & 148 & 101 & 7.98 & 4.51 & 10.82 & 73.7 & & 93.4 & 2,697 & 346 & 18.2 \\
\hline $10-31$ & 101 & 99 & 7.09 & 3.29 & 9.91 & 71.5 & 7.22 & 73.4 & 1,932 & 293 & 19.1 \\
\hline
\end{tabular}

Protocol: September 30, 1925, distemper first noticed. Dog slightly emaciated. October 27, profuse discharge from nose and eyes. Animal very weak. October 31, apparently improved. November 1, found dead. At autopsy, almost complete consolidation of right lower lobe and slight consolidation of left lower lobe were found. (No morphine used.)

dogs, as the drug causes a marked slowing of the heart. Anaemia was found in most of the animals but the degree was variable. Some of the dogs were much emaciated. For this reason the blood flows per kilo are higher than would be the case had they been well nour- 
ished. The emaciation was not of as great a degree as was the increase in cardiac output and a relatively small fraction of the increased blood flow per kilo can be attributed to undernutrition.

TABLE 9

Spontaneous preumonia

Animal V6. Weight varied 8.4 to 7.8 kilos

\begin{tabular}{l|c|c|c|c|c|c|c|c}
\hline Date & Pulse rate & $\begin{array}{c}\text { Temper- } \\
\text { ature } \\
\text { (Fahren- } \\
\text { heit) }\end{array}$ & $\begin{array}{c}\text { Arterial } \\
\text { oxygen }\end{array}$ & $\begin{array}{c}\text { Venous } \\
\text { oxygen }\end{array}$ & $\begin{array}{c}\text { Oxygen } \\
\text { consump- } \\
\text { tion per } \\
\text { minute }\end{array}$ & $\begin{array}{c}\text { Cardiac } \\
\text { output } \\
\text { per } \\
\text { minute }\end{array}$ & $\begin{array}{c}\text { Output } \\
\text { per } \\
\text { minute } \\
\text { per kilo }\end{array}$ & $\begin{array}{c}\text { Output } \\
\text { per beat }\end{array}$ \\
\hline & per minute & & $\begin{array}{c}\text { volumes } \\
\text { per cent }\end{array}$ & $\begin{array}{c}\text { volumes } \\
\text { ter cent }\end{array}$ & cc. & $c c$. & cc. & cc. \\
$10-12$ & 109 & 102.2 & 11.37 & 6.3 & 104.0 & 2,051 & 244 & 18.8 \\
$10-13$ & 110 & 102.0 & 11.59 & 5.94 & 91.5 & 1,622 & 196 & 14.7 \\
$10-18$ & 114 & 102.0 & 11.76 & 6.4 & 94.5 & 1,763 & 218 & 15.4 \\
$10-24$ & 118 & 102.6 & 12.33 & 7.76 & 104.4 & 2,276 & 284 & 18.4 \\
$11-2$ & 115 & 101.8 & 12.38 & 7.53 & 95.7 & 1,973 & 253 & 17.1 \\
\hline
\end{tabular}

Protocol: October 2, 1925, distemper first noticed. October 12, X-ray thought to show bilateral patchy pneumonia. October 18, animal appears slightly improved, less discharge from nose. October 24, X-ray shows clearing of apical shadows.

TABLE 10

Spontaneous pneumonia

Animal V12. Weight 11.4 kilos

\begin{tabular}{|c|c|c|c|c|c|c|c|c|c|c|}
\hline ڤัّ̆ & 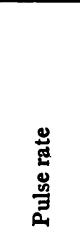 & 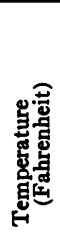 & 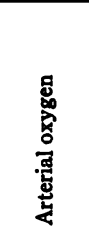 & 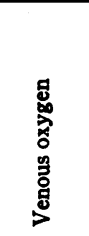 & 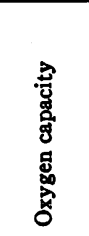 & 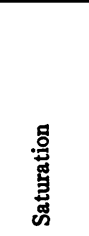 & 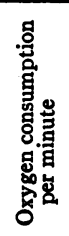 & 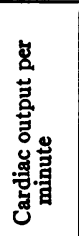 & 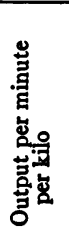 & 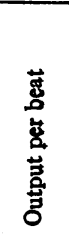 \\
\hline & $\underset{\text { minute }}{\text { per }}$ & & $\begin{array}{l}\text { oolumes } \\
\text { per cent }\end{array}$ & $\begin{array}{l}\text { oolumes } \\
\text { per cent }\end{array}$ & $\begin{array}{l}\text { volumes } \\
\text { per cent }\end{array}$ & Der cent & $c c$. & $c c$. & cc. & $c c$. \\
\hline $11-25$ & 68 & 100 & 14.55 & 11.16 & 16.10 & 90 & 79.1 & 2,363 & 207 & 34.7 \\
\hline $11-27$ & & & 14.60 & 11.28 & & & 81.4 & 2,420 & 212 & \\
\hline
\end{tabular}

Protocol: November 25, 1925, animal looks healthy. Temperature slightly elevated, blood flow very high. November 27, animal killed after experiment completed. Autopsy showed chronic broncho-pneumonia of nodular type. Very extensive pneumonia found on microscopic study.

Roentgenograms were taken in some instances and compared with plates from a normal group. The radiological findings were considerably less striking than the post mortem evidences of pneumonia. 
TABLE 11

Induced pneumonia

Animal M1. Weight varied 13.2 to 11.3 kilos

\begin{tabular}{|c|c|c|c|c|c|c|c|c|c|c|}
\hline$\underset{\varpi ّ}{S}$ & 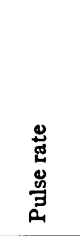 & 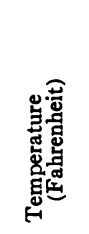 & 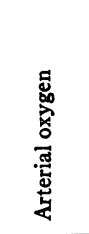 & 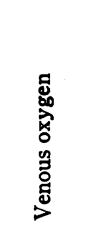 & 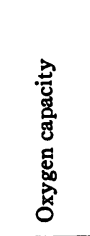 & 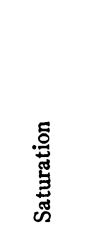 & 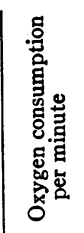 & 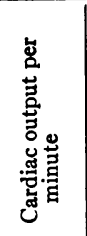 & 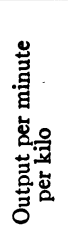 & 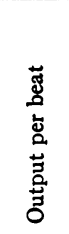 \\
\hline & $\underset{\text { minute }}{\text { per }}$ & & $\begin{array}{l}\text { volumes } \\
\text { per cent }\end{array}$ & $\begin{array}{l}\text { volumes } \\
\text { per cent }\end{array}$ & $\begin{array}{l}\text { volumes } \\
\text { per cent }\end{array}$ & per cent & $c c$. & $c c$. & $c c$. & $c c$. \\
\hline $5-26$ & 76 & 99.0 & 23.7 & 18.9 & 24.1 & 98.3 & 94.0 & 1,920 & 153 & 25.7 \\
\hline $5-30$ & 82 & 102.0 & 22.0 & 17.3 & 22.0 & 100.0 & 105.9 & 2,253 & 173 & 27.4 \\
\hline $6-7$ & 79 & 103.2 & 19.8 & 14.4 & 20.5 & 96.6 & 120.4 & 2,230 & 186 & 28.2 \\
\hline $6-18$ & 63 & 98.8 & 19.3 & 14.4 & & & 86.3 & 1,761 & 140 & 27.9 \\
\hline $6-20$ & 130 & 101.6 & 12.1 & 8.2 & 17.9 & 67.6 & 97.3 & 2,497 & 189 & 19.2 \\
\hline
\end{tabular}

(Figures in italics denote determinations done when animal was apparently normal; other figures denote determinations when pneumonia was thought to be present.)

Protocol: May 26, 1925, healthy dog. Control determination. May 30, second control determination. June 6 , inoculated with $40 \mathrm{cc}$. of 24 -hour growth Type II pneumococcus. June 7, temperature elevated, restless, looks well. June 18, apparently recovered. Inoculated again after determination. June 19, looks sick dyspneic, cyanotic, unable to stand. June 20, somewhat improved, given $20 \mathrm{cc}$. of 30 hour growth of Friedlander bacilli. June 21, condition unchanged. June 22, killed. Complete consolidation right middle and lower lobes, few scattered patches elsewhere. Findings confirmed by microscopic sections.

TABLE 12

Induced pneumonia

Animal M5. Weight varied 12 to 10.6 kilos

\begin{tabular}{|c|c|c|c|c|c|c|c|c|c|c|}
\hline ڤัँ & 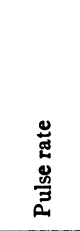 & 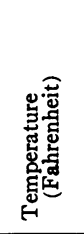 & 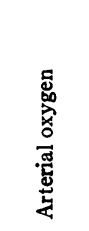 & 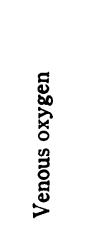 & 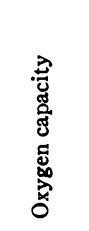 & 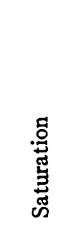 & 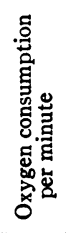 & 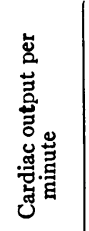 & 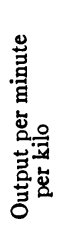 & 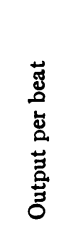 \\
\hline & $\begin{array}{c}\text { per } \\
\text { minute }\end{array}$ & & $\begin{array}{l}\text { volumes } \\
\text { per cent }\end{array}$ & $\begin{array}{l}\text { volumes } \\
\text { per cent }\end{array}$ & $\begin{array}{l}\text { volumes } \\
\text { per cent }\end{array}$ & per cent & $c c$ & $c c$. & $c c$. & $c c$. \\
\hline $5-30$ & 63 & 100.2 & 10.5 & 11.9 & 17.8 & 92.7 & 114.1 & 2,480 & 207 & 39.3 \\
\hline $6-2$ & 85 & 104.5 & 16.7 & 11.5 & 17.9 & 93.3 & 117.0 & 2,250 & 201 & 26.4 \\
\hline $6-4$ & 82 & 102.0 & 13.7 & 7.9 & 16.0 & 85.6 & 114.4 & 1,972 & 182 & 24.0 \\
\hline $6-5$ & 136 & 102.4 & 13.6 & 8.0 & 14.8 & 91.9 & 109.4 & 1,954 & 183 & 14.3 \\
\hline $6-8$ & 108 & 102.5 & 3.8 & 2.0 & 5.1 & 74.5 & 88.5 & 4,917 & 464 & 45.5 \\
\hline
\end{tabular}

(Figures in italics denote determinations done when animal was apparently normal; other figures denote determinations when pneumonia was thought to be present.)

Protocol: May 30, 1925, control studies. June 1, given $28 \mathrm{cc}$. of 24-hour growth Type I pneumococcus. June 2, looks healthy, not cyanotic, high fever. June 3, looks very sick. June 4, condition unchanged. June 8, animal is very weak. Autopsy performed after experiment was completed showed the lungs to be normal in the gross except for slight consolidation of both lower lobes. Microscopic sections show fairly extensive early broncho-pneumonia. 
Hydrogen ion concentrations were determined in six animals. Of these, four had extensive pneumonia with a high cardiac output

TABLE 13

Induced pneumonia

Animal V4. Weight varied 6.4 to 5.7 kilos

\begin{tabular}{l|c|c|c|c|c|c|c|c}
\hline Date & Pulse rate & $\begin{array}{c}\text { Temper- } \\
\text { ature } \\
\text { (Fahren- } \\
\text { heit) }\end{array}$ & $\begin{array}{c}\text { Arterial } \\
\text { oxygen }\end{array}$ & $\begin{array}{c}\text { Venous } \\
\text { oxygen }\end{array}$ & $\begin{array}{c}\text { Oxygen } \\
\text { consump- } \\
\text { tion per } \\
\text { minute }\end{array}$ & $\begin{array}{c}\text { Cardiac } \\
\text { output } \\
\text { per } \\
\text { minute }\end{array}$ & $\begin{array}{c}\text { Output per } \\
\text { minute } \\
\text { per kilo }\end{array}$ & $\begin{array}{c}\text { Output } \\
\text { per beat }\end{array}$ \\
\hline & per minute & & $\begin{array}{c}\text { volumes } \\
\text { per cent }\end{array}$ & $\begin{array}{c}\text { volumes } \\
\text { per cent }\end{array}$ & $c c$. & $c c$. & cc. & cc. \\
$12-4$ & 58 & 100.0 & 15.74 & 9.45 & 54.0 & 846 & 132 & 14.5 \\
$12-5$ & 88 & 98.0 & 16.96 & 10.65 & 58.9 & 933 & 155 & 10.6 \\
$12-7$ & 64 & 99.0 & 16.49 & 10.29 & 57.0 & 921 & 161 & 14.3 \\
$12-10$ & 100 & 102.2 & 13.51 & 7.91 & 60.4 & 1,079 & 189 & 10.7 \\
\hline
\end{tabular}

(Figures in italics denote determinations done when animal was apparently normal; other figures denote determinations when pneumonia was thought to be present.)

Protocol: December 4, 1925, control. studies. December 5, second control studies. After determination given $10 \mathrm{cc}$. of 19-hour growth Type III pneumococcus. December 7, does not look sick. December 10, somewhat dyspneic. Dog died of hemopericardium shortly after last puncture was made. At autopsy, the lung bases seemed heavy and the upper lobes normal. Microscopic sections show much congestion, extensive oedema, and fairly extensive leucocytic exudation. (Morphine 0.04 gram given before each determination.)

TABLE 14

Induced pneumonia

Animal V15. Weight 10.2 kilos

\begin{tabular}{c|c|c|c|c|c|c|c|c}
\hline Date & Pulse rate & $\begin{array}{c}\text { Temper- } \\
\text { ature } \\
\text { (Fahren- } \\
\text { heit) }\end{array}$ & $\begin{array}{c}\text { Arterial } \\
\text { oxygen }\end{array}$ & $\begin{array}{c}\text { Venous } \\
\text { oxygen }\end{array}$ & $\begin{array}{c}\text { Oxygen } \\
\text { consump- } \\
\text { tion per } \\
\text { minute }\end{array}$ & $\begin{array}{c}\text { Cardiac } \\
\text { output } \\
\text { per } \\
\text { minute }\end{array}$ & $\begin{array}{c}\text { Output per } \\
\text { minute } \\
\text { per kilo }\end{array}$ & $\begin{array}{c}\text { Output } \\
\text { per beat }\end{array}$ \\
\hline & per minute & & $\begin{array}{c}\text { volumes } \\
\text { per cent }\end{array}$ & $\begin{array}{c}\text { volumes } \\
\text { per cent }\end{array}$ & $c c$. & $c c$. & cc. & cc. \\
$12-14$ & 60 & 97.0 & 21.56 & 17.1 & 85.9 & 1,928 & 189 & 32.1 \\
$12-15$ & 84 & 100.2 & 18.06 & 13.86 & 76.4 & 1,819 & 178 & 21.6 \\
\hline
\end{tabular}

(Figures in italics denote determinations done when animal was apparently normal; other figures denote determinations when pneumonia was thought to be present.)

Protocol: December 14, control studies. Forty cubic centimeter of 18-hour growth Type III pneumococcus given after determination. December 15, listless and very weak. December 16, found dead. Autopsy revealed extensive consolidation of all lobes, confirmed by microscopic sections.

and two a less extensive pneumonia with normal blood flow. The $\mathrm{pH}$ values all fell within the range (720-735) found as normal for morphinized dogs by Harrison, Wilson and Blalock (11). 
TABLE 15

Induced Preumonia

Animal V2. Weight varied 13.6 to 11.4 kilos

\begin{tabular}{|c|c|c|c|c|c|c|c|c|c|c|c|}
\hline 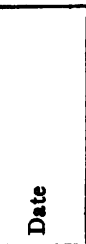 & 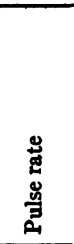 & 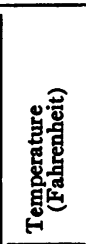 & 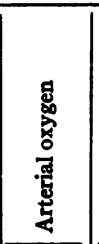 & 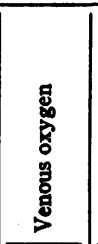 & 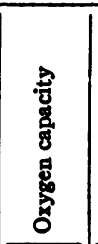 & 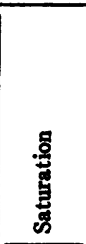 & 客 & 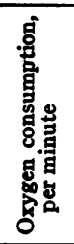 & 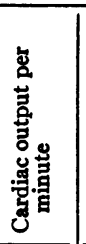 & 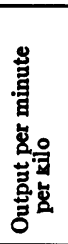 & 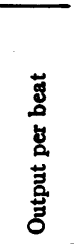 \\
\hline & minute & & volumes & volumes & $\begin{array}{l}\text { oolemeses } \\
\text { per cent }\end{array}$ & per cont & & $c c$. & & $c c$. & $c c$. \\
\hline $10-8$ & 67 & & 17.71 & 13.43 & 19.21 & 92.2 & & 85.2 & 1,990 & 146 & 29.7 \\
\hline $10-19$ & 71 & 98.0 & 17.70 & 13.25 & & & & 98.6 & 2,216 & 181 & 31.2 \\
\hline $10-22$ & & 100.0 & 17.45 & $|13.57|$ & & & & 82.6 & 2,123 & 174 & \\
\hline $10-29$ & 113 & 101.2 & 12.82 & 77.7 & 15.04 & 85.2 & 7.27 & 87.5 & 1,733 & 154 & 15.3 \\
\hline
\end{tabular}

(Figures in italics denote determinations done when animal was apparently normal; other figures denote determinations when pneumonia was thought to be present.)

Protocol: October 8, 19, and 22, control studies. October 23, X-ray shows normal lungs. October 26, $35 \mathrm{cc}$. of 10-hour growth Type I pneumococcus. October 27, slightly dyspneic, temperature 102 . October 29, weak, losing weight. Killed after determination. The left upper lobe was solid. Patches of broncho-pneumonia throughout rest of lungs. Microscopic sections showed extensive pneumonia.

TABLE 16

Induced pneumonia

Animal V5. Weight varied 5.9 to 5.6 kilos

\begin{tabular}{|c|c|c|c|c|c|c|c|c|c|c|}
\hline 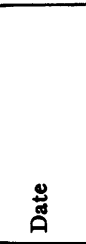 & 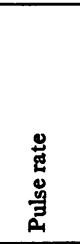 & 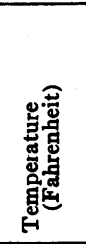 & 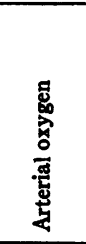 & 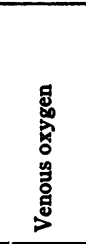 & 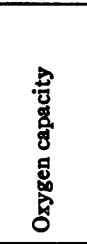 & 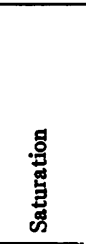 & 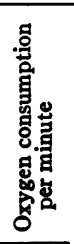 & 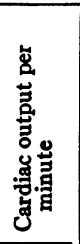 & 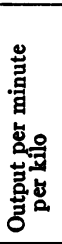 & 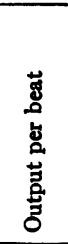 \\
\hline & $\begin{array}{c}\text { per } \\
\text { minute }\end{array}$ & & $\begin{array}{l}\text { volumes } \\
\text { per cent }\end{array}$ & $\begin{array}{l}\text { volumes ces } \\
\text { per cent }\end{array}$ & $\begin{array}{l}\text { volumes mes } \\
\text { per cont }\end{array}$ & per cent & $c c$. & $c c$ & $c c$. & $c c$. \\
\hline $10-25$ & 68 & 98.0 & 16.3 & 11.22 & & & 56.7 & 1,116 & 189 & 16.4 \\
\hline $10-26$ & 92 & 97.6 & 14.24 & 9.41 & 16.01 & 88.9 & $62: 6$ & 1.296 & 219 & 14.0 \\
\hline $10-29$ & 110 & 102.0 & 15.01 & 8.05 & & & 74.4 & 1,069 & 191 & 9.7 \\
\hline $11-1$ & 82 & 102.0 & 13.78 & 6.40 & 15.52 & 88.8 & 72.8 & 986 & 176 & 12.0 \\
\hline
\end{tabular}

(Figures in italics denote determinations done when animal was apparently normal; other figures denote determinations when pneumonia was thought to be present.)

Protocol: October 25, 1925, control study. X-ray showed normal lungs. October 26, second control study. After determination given $20 \mathrm{cc}$. of 10-hour growth Type I pneumococcus. October 27 , somewhat weak, slightly dyspneic, temperature 103. October 29 , condition unchanged. November 1 , improved, walking around, studies without morphine. Killed after determination. Autopsy showed few patches broncho-pneumonia both lower lobes. (Morphine, $\frac{3}{4}$ grain, given before each determination with the exception of the one on November 1.) 
Hastings, Neill, Morgan and Binger (12) found normal values for $\mathrm{pH}$ in pneumonic patients, whereas Leake, Vickers and Brown (13) found slight acidosis in the experimental pneumonia of dogs.

The animals developed a marked anaemia in many instances. The cause of this is not clear. Leake, Vickers, and Brown (13) found that in experimental pneumonia of dogs a great loss of circulating red blood cells occurred without compensatory regeneration. From our studies it is not possible to be sure that the diminution of oxygen was dependent entirely on loss of red blood corpuscles as counts were not made. It is possible that methemoglobin may have been present. If so the amount of methemoglobin was not significant, as no change was noted in the color of the aerated blood.

\section{DISCUSSION}

The results of the experiments here reported are discussed from two points of view.

1. The mechanism responsible for the increased output of the heart in pneumonia. Our present knowledge of the controlling factors of the circulation is comparable to our knowledge of the regulation of respiration before the classical observations of Zuntz (14) and Haldane and Priestley (15), demonstrating the importance of oxygen and carbon dioxide. A rational conception of the regulation of the cardiac output is of prime importance to clinicians as well as to physiologists. Therefore an attempt has been made to analyze the various factors which may have been responsible for the increased cardiac output in pneumonia. In an investigation now in progress these factors are being studied separately in an endeavor to ascertain their relationship to the circulation under less complicated conditions.

In pneumonia there are at least five abnormal conditions which may lead to alterations of the output of the heart. These are:

1. The effect of toxins produced by the infecting organisms on the tissues, producing fundamental disturbances of function

2. Acidosis (if present)

3. Increased metabolic rate

4. Anaemia

5. Lack of oxygen in the blood leaving the lungs 
The effect of toxins cannot be studied quantitatively with present methods, and so this possibility has been neglected in our further consideration.

Since Leake, Brown and Vickers found acidosis in dogs with pneumonia, and since Harrison, Wilson and Blalock found that acidosis produces an increase in the cardiac output of morphinized dogs, at-

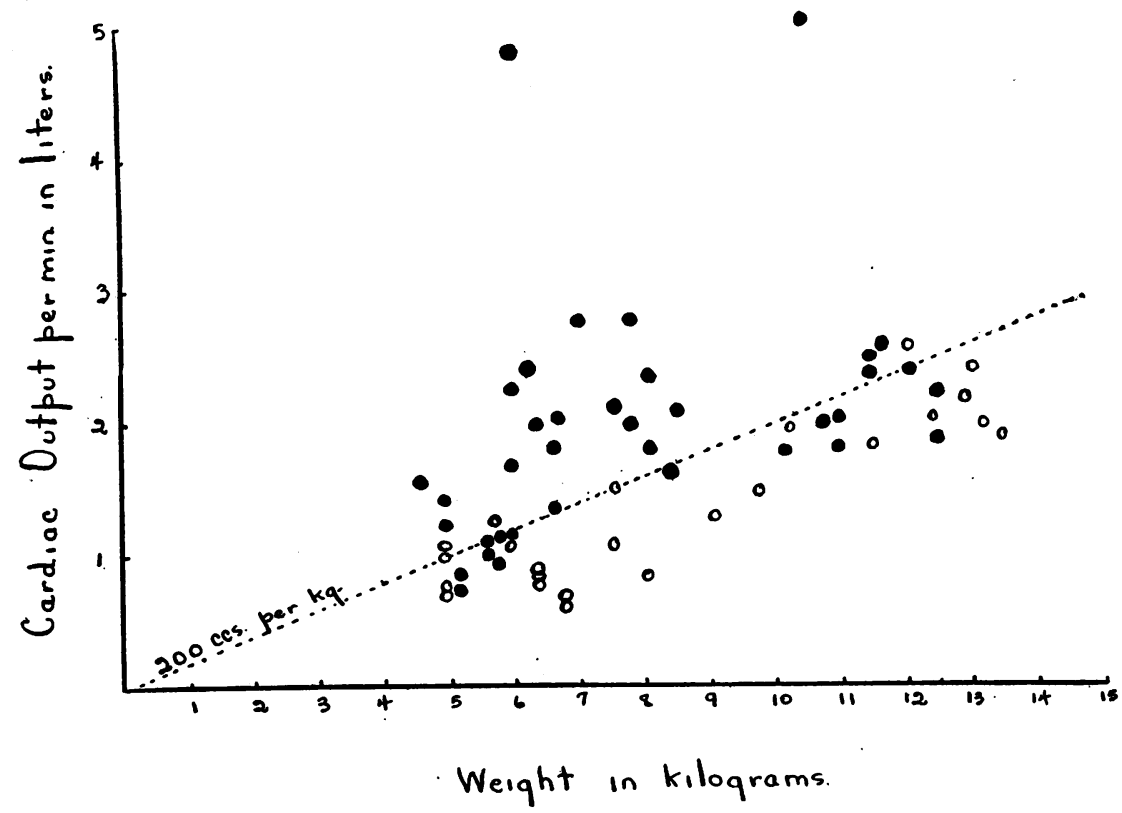

Fig. 1. The total cardiac output per minute is charted against the weights. The diagonal dotted line represents $200 \mathrm{cc}$. per minute per kilo of body weight. In this and the following figure the white circles denote normal dogs, the black circles-dogs with pneumonia. Nearly all the normal animals have a cardiac output of less than $200 \mathrm{cc}$. per kilo per minute, whereas most of the dogs with pneumonia have a higher output.

tention was first directed to this factor. However, as has been stated above, the $\mathrm{pH}$ value of the blood of the animals with pneumonia was found to fall within the same range as the blood $\mathrm{pH}$ value of normal dogs receiving similar doses of morphine. The $\mathrm{pH}$ decline necessary to produce a marked increase in cardiac output is greater than the degree of this morphine acidosis, as Harrison, Wilson and Blalock have shown. 
Satisfactory normal standards are not available for determining whether the basal metabolic rate of a dog is increased. Most of the dogs with pneumonia had some elevation of body temperature and an increased heat production may be assumed. The oxygen consumption per minute per kilo varied from 6 to $14 \mathrm{cc}$, and was in general in inverse ratio to the weight of the dog. In figure 2 a correlation is shown between the number of cubic centimeters of

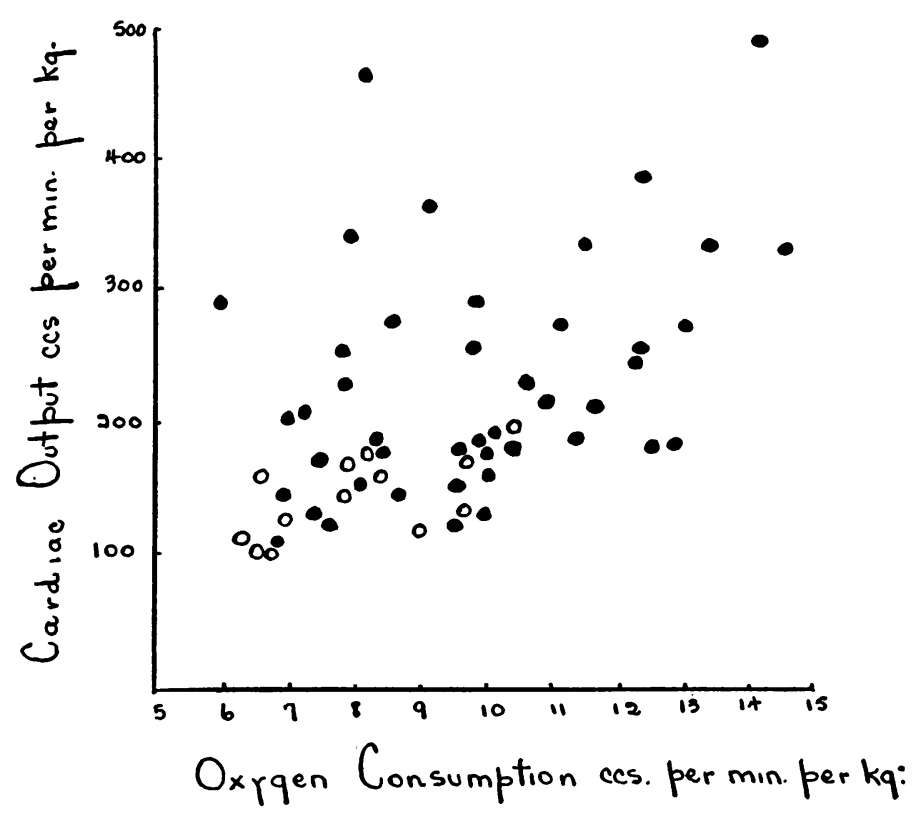

FIG. 2. The cardiac output per minute per kilo is charted against the oxygen consumed per minute per kilo. There is a general tendency for the cardiac output to be higher in the animals with a higher oxygen consumption, but this is not striking.

oxygen consumed per minute per kilogram and the cardiac output per minute per kilo. Although the higher values for the cardiac output coincide in a rough way with the higher values for oxygen consumption, the relationship is not striking.

A similar chart (fig. 3 ) depicts the correlation between the cardiac output and hemoglobin content (the values for hemoglobin were calculated from the oxygen capacities on the assumption that 20 
volumes per cent capacity equal 100 per cent hemoglobin). From this figure it appears that the relationship of increased output of the heart to anaemia is fairly definite, although caution is necessary in interpreting results when other complicating factors are present.

Cardiac output in relation to anoxemia is demonstrated in figure 4. Here the correlation is more suggestive than with the functions pre-

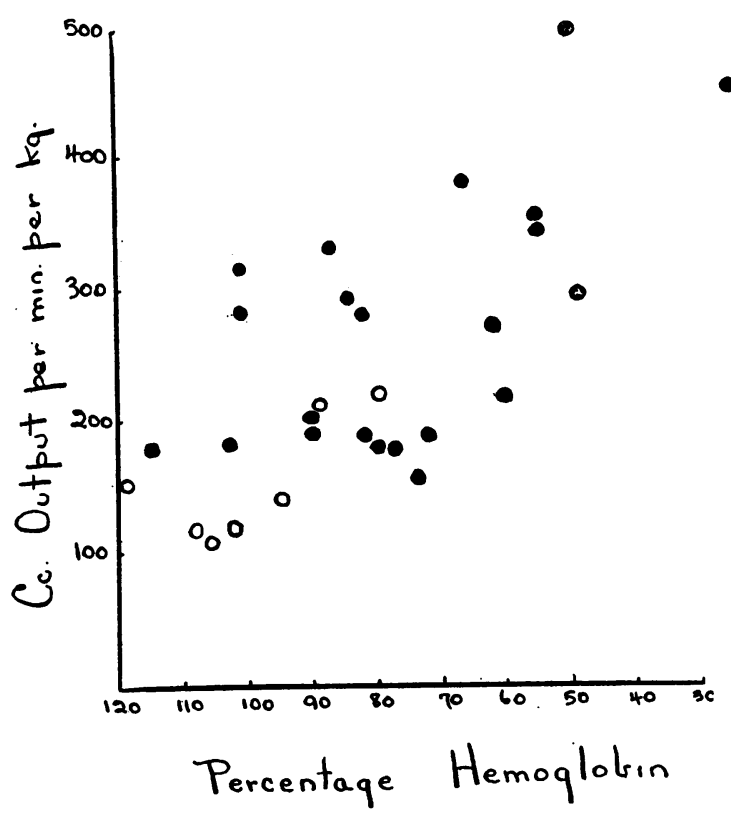

FIG. 3. The cardiac output per minute per kilo is charted against the percentage hemoglobin, which was calculated from the oxygen capacity on the assumption that 20 volumes per cent oxygen capacity equals 100 per cent hemoglobin. The reduction of hemoglobin is strikingly correlated with an increase in cardiac output.

viously plotted, but one is scarcely justified in drawing any definite conclusions under the complicated conditions of the experiments.

These results indicate that increased metabolic rate, anaemia of severe degree, and anoxemia may be of importance in regulating the blood flow. In order to evaluate each of these factors it is necessary that they be studied singly. This is being done at the present time and the results will be published at a later date. 
2. The clinical significance of an increased cardiac output in pneumonia. If one makes the assumption that the human heart responds to the pneumonic process in the same way as the heart of the rabbit (Leegaard) or dog-an assumption which seems likely but remains to be proved-certain probable deductions can be drawn.

$a$. When the infection is overwhelming and death comes in one to three days, there is little or no change in cardiac output. When the

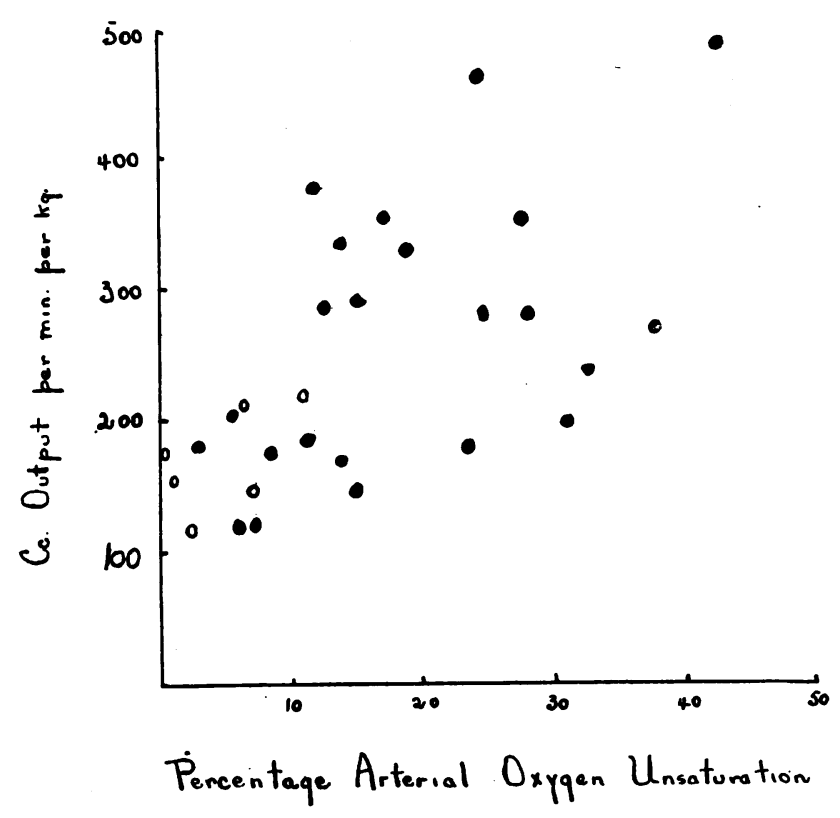

FIG. 4. The cardiac output per minute per kilo is charted against the percentage of arterial unsaturation-or degree of anoxemia. With high degrees of arterial unsaturation the cardiac output is greatly increased.

infection is mild there is relatively little change. The severe but not immediately overwhelming infection of several days standing apparently causes the greatest increase in blood flow. In these instances the cardiac output seems to stand in the same relation to the severity of the infection as does the leucocyte count.

$b$. The assumption that death occurs in pneumonia from cardiac failure seems to be true in one sense and false in another. The older conceptions of a heart muscle injured by toxin contracting more 
feebly seems incorrect. From the results of others, quoted above, and of this work, it is more logical to think of a heart doing more than the normal amount of work and failing eventually because of the increased load.

Of practical importance is the question as to whether the increased blood flow in pneumonia is a compensatory and hence necessary mechanism, or whether this increase is to be regarded as being per se a dangerous manifestation of the disease and leading eventually to failure of the circulation. It appears probable that both conceptions are correct. Blalock, Harrison and Wilson have shown experimentally that diminishing the minute ventilation by partially obstructing the respiration causes a great increase in the output of the heart. From their experiments they concluded that the circulation constitutes the second line of defense against failure of tissue metabolism and that the heart by increased output can compensate for an inefficient respiratory mechanism. In their experiments the $\mathrm{CO}_{2}$ elimination was markedly affected while relatively little change in the oxygen absorption was found. In the experiments on pneumonia reported in this paper the reverse is true, but the circulatory response is similar. We believe that either a rise of alveolar $\mathrm{CO}_{2}$ tension or a fall in alveolar oxygen tension, if of sufficient magnitude, will cause an increased cardiac output. The advantage to the body is obvious. The amount of oxygen taken up, or of $\mathrm{CO}_{2}$ given off per $1 \mathrm{cc}$. of blood in the lungs depends on the difference between the pressure of these gases in the alveoli and in the venous blood. If the alveolar $\mathrm{CO}_{2}$ tension rises, each unit of blood can give off less $\mathrm{CO}_{2}$. If the alveolar oxygen tension falls (beyond a certain level) each unit of blood can take up less oxygen. Hence, in either case, if the total exchange of gas is to remain constant more units of blood must pass through the lungs. The increased blood flow must be regarded therefore as a compensatory mechanism and an advantage, but obtained at the risk of possible cardiac failure. If the heart can carry the increased load until the infection is overcome, recovery may be expected; if the strain on the heart causes it to weaken, death results.

It is believed that these conceptions explain why circulatory failure occurs more rapidly in pneumonia than in other diseases, such as ty- 
phoid fever and acute miliary tuberculosis, comparable as to degree of "toxemia."

The relationship of anoxemia to the increased cardiac output has been discussed above, and severe anoxemia appears to be correlated with increased output of the heart. This affords a rational basis for the administration of oxygen in pneumonia. Increasing the alveolar oxygen tension should diminish the necessity for an increased cardiac output. If the heart of man reacts to pneumonia as does the heart of the dog, oxygen should have a very beneficial sedative action in those cases where cyanosis is marked.

In comparing the findings in experimental pneumonia with clinical pneumonia it is necessary to remember that the latter is frequently encountered in individuals with diseased hearts, whereas the experimental animals had normal hearts, so far as we could judge. It is possible that the pathological heart cannot increase its output to any considerable degree and this may account for the well known fact that pneumonia is more fatal in individuals suffering from cardiac diseases.

The value of "cardiac stimulants" remains an open question. Caffeine and camphor are commonly used. Aside from their value as respiratory stimulants, our purpose in giving them is to increase the output of the heart. If these drugs do not have this effect it is useless to give them. If they do have this effect, their value is questionable and possibly their use is harmful. Our knowledge of the circulation is too vague to permit an answer to the question of the advisability of increasing the work of an organ already working at or near its maximum level. Clinically one gains the impression that these drugs are of little value except in those patients who develop Cheyne-Stokes breathing. However, the "cardiac stimulants" are usually not given until the patient is well nigh moribund and therefore such an impression may not be correct. Further experiments and better methods for study of patients are needed before caffeine and camphor can be evaluated.

Digitalis is widely used in pneumonia. Cohn and Jamieson demonstrated that this disease does not alter the effect of the drug on the heart. 
Levy (16) has shown that digitalis diminishes the size of the heart shadow in pneumonia. In some work now in progress Leonard and Harrison have found that digitalis in therapeutic doses diminishes the output of the dog's heart. If we may believe that the dog's heart and human heart are comparable, one is led to assume that most patients with pneumonia need a cardiac sedative rather than a cardiac stimulant. Although the increased blood flow is probably compensatory, the heart must be protected against compensating to the extent of becoming weakened by the strain.

\section{SUMMARY}

The output of the heart of the dog has been studied by the Fick method. In pneumonia the cardiac output is usually increased. When the infection is of short duration and either mild or overwhelming, the increase is less than in the severe, well developed pneumonia of longer duration. The increase in cardiac output appears to bear little relation to changes in oxygen consumption, but appears to depend to some extent on anaemia and to a greater extent on anoxemia. On the assumption that the cardiac output is also increased in pneumonia in man, heart failure is to be ascribed to the increased strain on the circulation rather than to weakness of the myocardium. From this viewpoint oxygen and digitalis appear to be definitely indicated, whereas the value of caffeine and camphor is questionable.

It is a pleasure to express our appreciation to Dr. G. Canby Robinson and Dr. E. K. Marshall for advice, encouragement and many helpful suggestions in regard to this work.

\section{BIBLIOGRAPHY}

1. Newburgh, L. H., and Porter, W. T.: Jour. Exper. Med., 1917, xxii, 123.

2. Cohn, A. E., and Jamieson, R. A.: Jour. Exper. Med., 1917, xxv, 65.

3. Stadie, W. C.: Jour. Exper. Med., 1919, xxx, 215.

4. Leegaard, T.: Skand. Arch. f. Physiol., 1925, xlvi, 323.

5. Marshall, E. K.: Jour. Physiol., 1925, lxxii, 192.

6. Blalock, A., Harrison, T. R., and Wilson, C. P.: Archives of Surgery (in press).

7. Van Slyke, D. D., and Stadie, W. C.: Jour. Biol. Chem., 1921, xlix, 1.

8. Van Slyke, D. D., and Neill, J. M.: Jour. Biol. Chem., 1924, lxi, 523.

9. Hawkins, J. A.: Jour. Biol. Chem., 1923, lvii, 493. 
10. Cullen, G. E.: Jour. Biol. Chem., 1922, lii, 501.

11. Harrison, T. R., Wilson, C. P., and Blalock, A.: Jour. Clin. Investigation, 1925, i, 547.

12. Hastings, A. B., Neill, J. M., Morgan, H. J., and Binger, C. A. L.: Jour. Clin. Investigation, 1924, i, 25.

13. Leake, C. D., Vickers, J. L., and Brown, T. K.: Jour. Exp. Med., 1924, xxix, 303.

14. Luntz, H.: Archiv. Physiol., 1897, xlvi, 379.

15. Haldane, J. B., and Priestley, J. C.: Jour. Physiol., 1905, xxxii, 225.

16. Levy, R. L.: Arch. Int. Med., 1923, xxxii, 350. 